\title{
Sustained Release Nitric Oxide from Long Lived Circulating Nanoparticles
}

\author{
Pedro Cabrales ${ }^{1}$, George Han ${ }^{2}$, Camille Roche ${ }^{2}$, Parimala Nacharaju ${ }^{2}$, Adam J. \\ Friedman $^{2,3}$, and Joel M. Friedman ${ }^{2}$ \\ ${ }^{1}$ Department of Bioengineering, University of California, San Diego, La Jolla, CA \\ 2 Departments of Physiology and Biophysics, Albert Einstein College of Medicine, Bronx, NY \\ ${ }^{3}$ Division of Dermatology, Department of Medicine, Albert Einstein College of Medicine, Bronx, \\ NY
}

\begin{abstract}
The current limitations of nitric oxide (NO) delivery systems has stimulated an extraordinary interest in the development of compounds that generate NO in a controlled and sustained manner with a heavy emphasis on the treatment of cardiovascular disease states. This work describes the positive physiological response to the infusion of $\mathrm{NO}$ releasing nanoparticles prepared using a new platform based on hydrogel/glass hybrid nanoparticles. When exposed to moisture, these nanoparticles slowly release therapeutic levels of NO, previously generated through thermal reduction of nitrite to NO trapped within the dry particles. The controlled and sustained release of NO observed from these nanoparticles (NO-np) is regulated by its hydration over extended periods of time. In a dose-dependent manner, circulating NO-np both decreased mean arterial blood pressure and increased exhaled concentrations of $\mathrm{NO}$ over a period of several hours. Circulating NO-np induced vasodilatation and increased microvascular perfusion during their several hour circulation lifetime. Control nanoparticles (Control-np; without nitrite) did not induce changes in arterial pressure, although a decrease in the number of capillaries perfused and increase in leukocyte rolling and immobilization in the microcirculation was observed. The NO released by the NO-np prevents the inflammatory response observed after infusion of Control-np. These data suggest that NO release from NO-np is advantageous relative to other NO releasing compounds, because it does not depend on chemical decomposition or enzymatic catalysis; it is only determined by the rate of hydration. Based on the observed physiological properties, NO-np has clear potential as a therapeutic agent and as a research tool to increase our understanding of NO signaling mechanisms within the vasculature.
\end{abstract}

\section{Keywords}

Nanotechnology; Intravascular gas delivery; Cardiovascular; Hypertension; Vasodilation

Correspondence to: Pedro Cabrales, Ph.D., Bioengineering, University of California, San Diego, 9500 Gilman Drive, Mail code 0412, La Jolla, CA 92093, Telephone: (858) 534-2315, pcabrales@ucsd.edu.

Publisher's Disclaimer: This is a PDF file of an unedited manuscript that has been accepted for publication. As a service to our customers we are providing this early version of the manuscript. The manuscript will undergo copyediting, typesetting, and review of the resulting proof before it is published in its final citable form. Please note that during the production process errors may be discovered which could affect the content, and all legal disclaimers that apply to the journal pertain. 


\section{Introduction}

Nitric oxide (NO) is a lipophilic, diatomic, free radical which is surprisingly stable and soluble in aqueous solutions when compared to other radical species [1]. Endogenous NO is produced enzymatically via $\mathrm{L}$-arginine conversion to NO by three distinct NO synthase (NOS) pathways [2]. With respect to vascular function, shear stress exerted on the luminal (endothelial) surface stimulates vascular endothelial NOS (eNOS), regulating numerous vascular functions, principally smooth muscle tension [3]. Intravascular NO supplementation has many complications as well as is short lived due to the rapid scavenging rate of NO by hemoglobin within erythrocytes [4]. Currently, the clinical therapeutic potential of NO has only been exploited via inhaled NO gas from pressurized tanks. While this approach is inconvenient and costly [5], inhaled NO gas is still the preferred and only approved NO treatment for acute pulmonary hypertension [6]. Other alternatives for intravascular NO therapy include formulations based on compounds containing either $\mathrm{NO}$ or an $\mathrm{NO}$ precursor in a stable form, which typically lack the capacity for controlled and sustained delivery [7]. Thus, despite the considerable therapeutic potential of NO, systemic deployment of NO to the bedside has proven very difficult.

Our current platform utilizes a sol-gel precursor in conjunction with a glass-forming combination of chitosan and polyethylene glycol to form a fine powder comprised of nanoparticles upon drying via lyophilization (Figure 1A). This hydrogel-glass hybrid material retains $\mathrm{NO}$ or an NO precursor in a stable forms when dry, and release NO upon exposure to moisture. This release process is controlled by the amount and characteristics of the materials used with drastic differences in NO release kinetics obtainable based on modification of described components [8]. These nanoparticles suffer minimal changes of release properties during long-term dry storage. The nanoparticles, seen on transmission electron microscopy (Figure 1B), are formed spontaneously and average $10 \mathrm{~nm}$ in diameter. Since a thermally driven redox reaction involving nitrite is used to generate the NO within the particles, the formed NO is retained within the dry particles [8].

The largest family of NO donors currently in use is NONOates, also known as diazeniumdiolates. They are complexes of $\mathrm{NO}$ with nucleophiles, for which decomposition rate depends on $\mathrm{pH}$, temperature, and the nature of the nucleophile. $[9,10]$ To compare the action of the NO-np platform to established NO donors, two particular NONOates were selected and examined based on their disparate, but physiologically relevant, decomposition rates (DETA NONOate, half-life of 20 hours at $37^{\circ} \mathrm{C}, \mathrm{pH} 7.4$; and DPTA NONOate, halflife of 3 hours $37^{\circ} \mathrm{C}, \mathrm{pH}$ 7.4).[7,11]

In this paper we provide in vivo experimental evidence and the conceptual framework through which to analyze intravascular exogenous supplementation of NO. Given the critical importance of $\mathrm{NO}$ in regulating pathophysiologic states involving the vasculature, controlled and sustained intravascular NO delivery could profoundly impact current treatment of cardiovascular disease states. This work presents physiological responses to circulating NO releasing hybrid hydrogel/glass nanoparticles (NO-np). In this system, hydration controls the release of $\mathrm{NO}$ generated through thermal reduction of nitrite trapped within the dry particles [8].

The present investigation describes how a novel redox chemistry in solid matrices can be used as the basis for new platforms to generate powder formulations that are not only capable of delivering NO in a sustained fashion but also are straightforward to store and apply. Here we show that in a dose-dependent manner, circulating NO-np increased exhaled NO, decreased blood pressure, and induced microvascular dilatation over several hours without inducing an inflammatory response. Conversely, control nanoparticles (made 
without nitrite, therefore lacking NO release) did not produce hemodynamic changes. The sole observed adverse effect from circulating NO-np was methemoglobin formation, inherent to NO reaction with hemoglobin. NO-np physiological responses clearly suggest their potential as a therapeutic agent for cardiovascular, inflammatory, and thrombotic disorders and to promote a more in-depth understanding of NO signaling mechanisms.

\section{Material and Methods}

\section{Synthesis of NO-np}

The synthesis of NO-np was recently reported.[8] Briefly, a hydrogel/glass composite was synthesized using a mixture of tetramethylorthosilicate, polyethylene glycol, chitosan, glucose, and sodium nitrite in a $0.5 \mathrm{M}$ sodium phosphate buffer $(\mathrm{pH} 7)$. Control nanoparticles were produced identically, but without nitrite. The nitrite was reduced to NO within the matrix because of the glass properties of the composite effecting redox reactions initiated with thermally generated electrons from glucose. After redox reaction, the ingredients were combined and dried using a lyophilizer, resulting in a fine powder comprising nanoparticles containing NO. Once exposed to an aqueous environment, the hydrogel properties of the composite allow for an opening of the water channels inside the particles, facilitating the release of the trapped NO over extended time periods.

\section{Transmission electron microscopy}

Transmission electron microscopy was used to characterize morphology of the nanoparticles. Samples were mixed with $1 \%$ Uranyl Acetate, placed onto a freshly glowed carbon coated grid, and blotted dry. Imaging was performed with a JEOL 100CX II transmission electron microscope (JEOL Ltd., Tokyo, Japan) operating at 80kV.

\section{Animal Preparation}

Investigations were performed in 50 - $65 \mathrm{~g}$ male Golden Syrian Hamsters (Charles River Laboratories, Boston, MA) fitted with a dorsal skinfold chamber window. Animal handling and care followed the NIH Guide for the Care and Use of Laboratory Animals. All experimental protocols were approved by the local animal care committee. The hamster chamber window model is widely used for microvascular studies in the unanesthetized state, and the complete surgical technique is described in detail elsewhere.[12]

\section{Inclusion Criteria}

Animals were suitable for the experiments if: 1) systemic parameters were within normal range, namely, heart rate $(\mathrm{HR})>340$ beat $/ \mathrm{min}$, mean arterial blood pressure (MAP) $>80$ $\mathrm{mmHg}$, systemic Hct $>45 \%$, and arterial oxygen partial pressure $\left(\mathrm{PaO}_{2}\right)>50 \mathrm{mmHg}$; and 2) microscopic examination of the tissue in the chamber observed under $650 \times$ magnification did not reveal signs of edema or bleeding. Hamsters are a fossorial species with a lower arterial $\mathrm{PO}_{2}$ than other rodents due to their adaptation to the subterranean environment.

\section{NO-donors or NO-nanoparticles infusion}

Animals were infused with NO releasing agents suspended in deoxygenated saline. Solutions were infused in a volume of $50 \mu \mathrm{l}$ (equivalent to less than $2 \%$ of the animals blood volume) via the jugular vein at a rate of $100 \mu \mathrm{l} / \mathrm{min}$. The following NO donors (Cayman Chemicals, Ann Arbor, MI) were used: DETA-NONOate (Diethylenetriamine NONOate, CAS 146724-94-9) and DPTA-NONOate (Dipropylenetriamine NONOate, CAS 146724-95-0). 


\section{Systemic Parameters and Blood Chemistry}

MAP and heart rate (HR) were recorded continuously (MP 150, Biopac System; Santa Barbara, CA). Hct was measured from centrifuged arterial blood samples taken in heparinized capillary tubes. Hb content was determined spectrophotometrically from a single drop of blood (B-Hemoglobin, Hemocue, Stockholm, Sweden). The methemoglobin (MetHb) level was measured via the cyanomethemoglobin method.[13] Arterial blood was collected in heparinized glass capillaries and immediately analyzed for $\mathrm{PaO}_{2}, \mathrm{PaCO}_{2}$, base excess (BE) and pH (Blood Chemistry Analyzer 248, Bayer, Norwood, MA). The comparatively low $\mathrm{PaO}_{2}$ and high $\mathrm{PaCO}_{2}$ of these animals is a consequence of their adaptation to a fossorial environment.

\section{Plasma nitrite/nitrate}

Blood samples were collected from carotid artery catheter and centrifuged to separate RBCs and plasma. Plasma proteins were removed by adding equivolume of methanol, and centrifuged at $15000 \mathrm{rpm}$ for $10 \mathrm{~min}$ at $4^{\circ} \mathrm{C}$. Concentration of nitrite and nitrate in the supernatant were measured with a NOx analyzer (ENO-20; Eicom, Kyoto, Japan). This analyzer combines Griess method and high-performance liquid chromatography.

\section{Exhaled NO}

Gaseous NO levels were measured using a chemiluminescent NO analyzer (Sievers NO analyzer, Model 280i, Boulder, CO). Pure air at $1 \mathrm{~L} / \mathrm{min}$ was continuously supplied via a modified sealed tube similar to that used to secure the hamster to the microscope during microvascular studies. $0.2 \mathrm{~L} / \mathrm{min}$ were introduced into the $\mathrm{NO}$ analyzer for one minute every ten minutes, while excess flow was evacuated through an underwater seal $\left(1 \mathrm{~cm} \mathrm{H}_{2} \mathrm{O}\right)$. $\mathrm{NO}$ calibration gases were introduced at the location where the animals sit during the experiment, to account for reactions between oxygen and NO.

\section{Total NO released (NO agents decomposition)}

Gaseous NO levels were measured using a chemiluminescent NO analyzer (Sievers NO analyzer, Model 280i, Boulder, CO). NO donors or NO-np were added to Di water (5 ml). pH was balanced using Trizma Base and Trizma HCI (Sigma-Aldrich, Saint Louis, Missouri). Solutions were continuously bubbled with pure nitrogen $(0.2 \mathrm{~L} / \mathrm{min})$. The gas phase was collected into the $\mathrm{NO}$ analyzer for a minute every set period of time, and the excess flow was evacuated through an underwater seal $\left(1 \mathrm{~cm} \mathrm{H}_{2} \mathrm{O}\right)$.

\section{Microvascular Experimental Setup}

The unanesthetized animal was placed in a restraining tube with a longitudinal slit from which the window chamber protruded, then fixed to the microscopic stage of a transillumination intravital microscope (BX51WI, Olympus, New Hyde Park, NY). The animals were given $20 \mathrm{~min}$ to adjust to the change in the tube environment before measurements. The tissue image was projected onto a CCD camera (COHU 4815) connected to a video recorder and viewed on a monitor. Measurements were carried out using a 40× (LUMPFL-WIR, numerical aperture 0.8 , Olympus) water immersion objective. Microhemodynamic measurements were compared to baseline levels obtained before the experimental procedure. The same vessels and functional capillary fields were followed so that direct comparisons to their baseline levels could be performed allowing for more robust statistics for small sample populations.

\section{Microhemodynamics}

Arteriolar and venular blood flow velocities were measured on-line by using the photodiode cross-correlation method (Photo Diode/Velocity Tracker Model 102B, Vista Electronics, 
San Diego, CA). The measured centerline velocity was corrected according to vessel size to obtain the mean $\mathrm{RBC}$ velocity $(\mathrm{V})$. A video image-shearing method was used to measure vessel diameter (D). Blood flow (Q) was calculated from the measured values as $\mathrm{Q}=\pi \times \mathrm{V}$ $(\mathrm{D} / 2)^{2}$. Changes in arteriolar and venular diameter from baseline were used as indicators of a change in vascular tone. This calculation assumes a parabolic velocity profile and has been found to be applicable to tubes of $15-80 \mu \mathrm{m}$ internal diameters and for Hcts in the range of $6-60 \%$.

\section{Functional Capillary Density (FCD)}

Functional capillaries, defined as those capillary segments that have RBC transit of at least a single $\mathrm{RBC}$ in a $45 \mathrm{~s}$ period, in 10 successive microscopic fields were assessed, totaling a region of $0.46 \mathrm{~mm}^{2}$. Each field had between two and five capillary segments with RBC flow. FCD $\left(\mathrm{cm}^{-1}\right)$, i.e., total length of RBC perfused capillaries divided by the area of the microscopic field of view, was evaluated by measuring and adding the length of capillaries that had RBC transit in the field of view. The relative change in FCD from baseline levels after each intervention is indicative of the extent of capillary perfusion.

\section{Leukocyte-endothelium interaction}

Intravenous injection of acridine orange ( $5 \mathrm{mg} / \mathrm{kg}$ solution in saline) was used to quantify microvascular leukocytes (adherence and rolling) in post capillary venules $(25-50 \mu \mathrm{m})$. Low light fluorescent microscopy was used during leukocyte endothelium assessment (ORCA 9247, Hamamatsu, Tokyo, Japan). Briefly, the straight portion of venules was exposed to low intensity epi-illumination for $60 \mathrm{~s}$ and digitally video recorded (10 frames/s). Leukocytes were counted during video playback in a $100 \mu \mathrm{m}$ length segment and categorized according to their flow behavior as "passers," including "free-flowing" leukocytes, and "rolling" and "immobilized" cells.[14] Measurements of immobilized leukocytes were measured over a period of 120 minutes, as acridine orange toxicity affects leukocyte viability.

\section{Circulating time of NO-np}

NO-np were incubated with Rhodamine 6G (20mg/dl, Sigma-Aldrich. St. Louis) for $30 \mathrm{~min}$, then washed three times and re-suspended in saline, and infused IV as described previously. [15] Characterization of the nanoparticles showed that they could be synthesized in the nanometer range with high photostability and minimal dye leakage. A photometric assembly in an Olympus BX51WI was used to determine fluorescence of particles in the microvascular window preparation. Briefly, tissue was illuminated $(525 \mathrm{~nm})$ using a ALPHA Vivid XF105-2 (Omega Filters, Brattleboro, VT) and fluorescence $(555 \mathrm{~nm}$ ) was evaluated by using a photomultiplier (Hamamatsu R928, Tokyo, Japan). Output from the photomultiplier was calibrated in terms of the number of detected photons per unit area of emission.

\section{Drugs NO and Guanylate Cyclase metabolism}

NO synthase inhibition was induced by continuous infusion of L-NAME (N-nitro-Larginine methyl ester, 30mg/kg, 20 $\mu 1 / \mathrm{min}$; Sigma-Aldrich. St. Louis, MO). Irreversible heme-site inhibitor of soluble guanylyl cyclase was induced with ODQ $(1 \mathrm{H}-$ [1,2,4]Oxadiazolo[4,3-a]quinoxalin-1-one; (Cayman Chemicals, Ann Arbor, MI) by IV infusion of $2 \mathrm{mg} / \mathrm{kg} 20 \mathrm{~min}$ before infusion of NO-np.[16] Selective inhibition of cGMPspecific phosphodiesterase was produced by continuous infusion of Zaprinast (2-(oPropoxyphenyl)-8-azapurin-6-one; CAS 37762-06-4; $0.1 \mathrm{mg} / \mathrm{ml} / \mathrm{hr}$ ). Zaprinast enhances the vasodilatory effects of $\mathrm{NO}$ in a range of vascular tissues by prolonging the cGMP-mediated activation of cGMP-dependent protein kinase.[17] 


\section{Data analysis}

Results are presented as mean standard deviation. Data within each group were analyzed using analysis of variance for repeated measurements (ANOVA, Kruskal-Wallis test). When appropriate, post hoc analyses were performed with the Dunns multiple comparison test. All statistics were calculated using GraphPad Prism 4.01 (GraphPad Software, Inc., San Diego, $\mathrm{CA})$. Changes were considered statistically significant if $\mathrm{P}<0.05$.

\section{Results}

\section{Total NO release from NONOate and NO-np}

A NONOate theoretically releases 2 moles of NO per mole of parent compound, although experimentally at $37^{\circ} \mathrm{C}$ and $\mathrm{pH} 7.4$, DETA NONOate liberates $10.7 \mu \mathrm{mols}$ of $\mathrm{NO} / \mathrm{mg}$ and DPTA NONOate liberates $9.4 \mu \mathrm{mols}$ of NO/mg. In contrast to the NONOates, the NO releasing nanoparticle (NO-np) utilizes a novel redox chemistry to generate and capture electrons, reducing nitrite to NO and retaining it within the particles. NO-np half-life in vitro

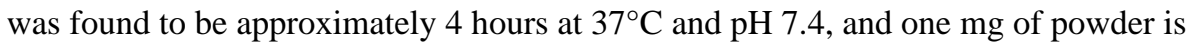
capable of releasing $0.32 \mu \mathrm{mols}$ of $\mathrm{NO}$ (Figure $1 \mathrm{C}$ ). Importantly, in vitro one $\mathrm{mg}$ of NONOates released more than 30 times more NO compare to NO-np. When suspended at lower $\mathrm{pH}$ levels, the total mount of $\mathrm{NO}$ released did not change, but faster rates of release were measured (Figure 1 C).

A total of 110 animals were entered into the study; all animals tolerated all of the protocols without visible signs of discomfort. Four sets of experiments were performed: i) changes in blood gas parameters, systemic hemodynamics (blood pressure and heart rate) and microhemodynamics (microvessel diameter and blood flow); ii) microvascular inflammatory parameters (Leukocyte activation: immobilized and rolling); iii) exhaled NO and circulating time; and, iv) NOS and cGMP methabolism. Five animals per group were assigned to each set experiments. All groups were statistically similar $(\mathrm{P}>0.40)$ in systemic and microcirculation parameters at baseline.

\section{Blood gas parameters}

Table 1 presents summary of blood gas parameters at baseline and after infusion of NO-np and Control-np. No changes in Hct and $\mathrm{Hb}$ were measured after infusion of the NO-np or Control-np. Infusion of NO-np increased MetHb levels from baseline after $2 \mathrm{~h}$. MetHb levels $4 \mathrm{~h}$ after infusion of NO-np were $9 \pm 2 \%$ for $10 \mathrm{mg} / \mathrm{kg}$ and $14 \pm 3 \%$ for $20 \mathrm{mg} / \mathrm{kg}$, respectively. Plasma nitrite and nitrate increased from baseline after infusion of NO-np proportional to the dose of NO-np infused at $1 \mathrm{~h}$ and $2 \mathrm{~h}$ after infusion. Arterial blood $\mathrm{pO}_{2}$ increased from baseline after infusion of NO-np. Arterial blood $\mathrm{pH}$ decreased from baseline compared after infusion of NO-np. Consequentially, blood acid-base balance decreased from baseline after infusion of NO-np. No significant change in arterial blood gas parameter was measured after infusion of Control-np.

\section{Systemic parameters}

MAP decreased after infusion of NO-np in a dose dependent fashion (Figure $2 \mathrm{~A}, \mathrm{~B}$ ). Maximal decrease in MAP in animals treated with NO-np was measured at 90 min after infusion. MAP restored to baseline after $3 \mathrm{~h}$ after infusion of NO-np. MAP increased above baseline in animals treated with NO-np at $3.5 \mathrm{~h}$ after infusion. MetHb levels $4 \mathrm{~h}$ after infusion of NO-np were $9 \pm 2 \%$ for $10 \mathrm{mg} / \mathrm{kg}$ and $14 \pm 3 \%$ for $20 \mathrm{mg} / \mathrm{kg}$, respectively. Infusion of control particles did not change MAP over the $4 \mathrm{~h}$ observation period. HR changes after infusion of NO-np were inversely correlated with the changes in MAP. 
Infusion of identical amounts of DETA NONOate and DPTA NONOate produced similar decreases in blood pressure (Figure $2 \mathrm{C}, \mathrm{D}$ ). The impact on vascular tone following NONOates were inefficient compare to NO-np, based on the in vitro NO release studies. The physiologic impact of the higher doses of NO provided by NONOate is manifested by a significant increase on metHb formation with a subsequent decrease in hemoglobin oxygen carrying capacity. MetHb levels $4 \mathrm{~h}$ after infusion of DPTA NONOate were $19 \pm 3 \%$ for 10 $\mathrm{mg} / \mathrm{kg}$ and $24 \pm 3 \%$ for $20 \mathrm{mg} / \mathrm{kg}$, respectively. MetHb levels $4 \mathrm{~h}$ after infusion of DETA NONOate were $21 \pm 3 \%$ for $10 \mathrm{mg} / \mathrm{kg}$ and $26 \pm 4 \%$ for $20 \mathrm{mg} / \mathrm{kg}$, respectively.

\section{Exhaled NO after infusion of NO-np}

Exhaled NO increased after infusion of NO-np, maximal exhaled NO levels were measured $1 \mathrm{~h}$ after infusion (Figure $3 \mathrm{~A}$ ). Increased exhaled NO levels were maintained over a period of $2 \mathrm{~h}$, after that exhaled NO decreased. Exhaled NO levels in animals treated with Controlnp did not increase.

\section{Circulation time}

Intravascular fluorescence is proportional to the concentration of circulating NO-np (Figure $3 \mathrm{~B}$ ). Infusion of fluorescently label nanoparticles produced an increase in intravascular fluorescence. Circulating particles were observed up to 6 hours after infusion. Although, no evidence of fluorescent labeled NO-np was observed by the next day. Circulating labeled NO-np were observed up to $6 \mathrm{~h}$ after infusion, but not 24 hours following intravenous introduction. However, control-np were not observed in the circulation 4 hours after infusion.

\section{Hemodynamics parameters}

Circulating NO-np produced microvascular vasodilatation and increased blood flow compared to baseline and Control-np (Figure $4 \mathrm{~A}, \mathrm{~B}$ ). Infusion of control particles did not affect arteriolar diameter or blood flow. In the venular side no significant change in diameter was measured after infusion of NO-np or Control-np. Venular blood flow was reduced from baseline after infusion of $20 \mathrm{mg} / \mathrm{kg}$ NO-np. Functional capillary density (FCD) was not affected in animals treated with NO-np, however control nanoparticles reduced the FCD (Figure 4 C).

\section{Leukocyte activation parameters}

Number of immobilized leukocytes increased after infusion of control particles at $1 \mathrm{~h}$ and $2 \mathrm{~h}$, respectively (Figure 5 A,B,C). NO-np did not increase immobilized leukocytes compared to baseline, although animals treated with $20 \mathrm{mg} / \mathrm{kg}$ demonstrated a higher number of immobilized leukocytes as compared to animals treated with $10 \mathrm{mg} / \mathrm{kg}$. Number of rolling leukocytes increased from baseline after infusion of Control-np at all time points. NO-np did not increase the number of rolling leukocytes compared to baseline, and no difference was measured between doses of NO-np.

\section{NO synthase and GMP metabolism (10 $\mathrm{mg} / \mathrm{kg}$ )}

Infusion of NO-np during NO synthase inhibition with L-NAME, partially reversed the resulting vasoconstriction and increase in MAP as compared to Control-np (Figure $6 \mathrm{~B}$ ). Infusion of $10 \mathrm{mg} / \mathrm{kg}$ of NO-np during irreversible inhibition of heme-site soluble guanylyl cyclase with ODQ resulted in minimal vascular impact, slightly but significantly decreasing the vasoconstriction and hypertension induced by ODQ (Figure 6 C). Infusion of NO-np and Control-np after treatment with the phosphodiesterase inhibitor Zaprinast, increased the vasodilatory effects of the NO-np (Figure 6 D). 


\section{Discussion}

The principal finding is that the physiological response to the infusion of NO-np in the circulatory system is consistent with a sustained release of physiologically relevant concentrations of NO. This sustained release is reflected in both the decrease in blood pressure and the increase in vascular relaxation, compared to the absence of any vascular response following the infusion of the Control-np. The efficacy and potential potency of the exogenous NO released by the NO-np is evident from the systemic hypotension and increase in exhaled NO induced by infusion of $20 \mathrm{mg} / \mathrm{kg}_{\mathrm{bw}}$. Additionally, the released NO inhibited the intravascular inflammatory response observed with the Control-np, suggesting an immunomodulatory role for this technology. The levels of exhaled NO and modest increase in nitrite levels are not compatible with a scenario in which nitrite is first released and then converted to NO. Furthermore, under the non-acidic physiological conditions there should be minimal NO formation from any released nitrite.

From the physiological viewpoint, exogenous NO released in the intravascular compartment by the NO-np will be partially scavenged by the RBC hemoglobin, and only a portion diffuses abluminally. A minor increase in intra-luminal $\mathrm{NO}$ concentration affects the endothelial NO diffusion gradients, thereby allowing the endothelial derived NO to diffuse further from cell to cell, independent of receptors and channels. The consequence of the high reactivity and short half-life of $\mathrm{NO}$ is that its biological action and impact are determined primarily by the rate of formation and the diffusion gradients at its site of action. Generation and transport of NO in vivo are closely linked to endothelial cell function, local shear stress, and local oxygen conditions. All of these factors pose a major challenge for systemic NO delivery based on using inhaled gaseous NO delivered from a pressurized tank. This mode of NO delivery which is neither convenient nor cost effective is also potentially harmful given the initial high local concentrations. Conversely, NO-np are stable, easily deployed materials that release pure, biologically relevant non-toxic concentrations of NO in a controllable sustained manner. Pulmonary microcirculation appears to be affected by the NO-np, as the arterial blood $\mathrm{pO}_{2}$ increased from baseline after infusion of NO-np, as compared to control particles. Studies with other NO donors indicated that these drugs are generally less potent on intralobar pulmonary arteries (resistance vessels) than on main pulmonary arteries (conduit vessels).[18,19] However, whole animal studies do not allow us to differentiate between resistance and conduit pulmonary arteries in this study.

Infusion of NO-np and NONOates decreased blood pressure in a dose dependent fashion, and control-np did not have effect on blood pressure. However, NONOates seem highly inefficient when compared to NO-np, as 30 times more NO release from NONOates was required to produce similar physiologic impact. Therefore, administration of NONOates induced higher methemoglobin formation than NO-np, with subsequent less effect on oxygen carrying capacity. However, a sustained increase in intra-luminal $\mathrm{NO}$ affects the endothelial NO diffusion gradients, thus allowing the endothelial derived NO to diffuse further. Reflecting sustained generation/distribution of NO from NO-np, exhaled NO levels increased for several hours after infusion with maximum levels achieved $1 \mathrm{~h}$ after infusion. Microvascular vasodilatation data suggest that the release of NO accounts for vasorelaxant responses produced by the NO-np, as the control nanoparticles did not induce relaxation at corresponding concentrations.

The exact chemical processes within the nanoparticle to explain NO formation from nitrite are not yet clearly established. Initial work describing the nanoparticle platform assumed that the NO was formed in the sugars, such as trehalose, because they support efficient electron transfer over macroscopic distances where either thermal or photo initiated processes are used to start the electron transfer [8]. It has been clearly demonstrated that 
thermal initiation of electron transfer generated within a sugar-glass can be used to bring about thermal reduction of either ferric hemeproteins $[20,21]$ or nitrite. $[8,22,23]$ In the case of nitrite it is clear that $\mathrm{NO}$ is being formed and trapped within the glass. When the glass "melts" upon exposure to water, the NO is rapidly released [8]. This process may be responsible for the formation of NO from nitrite within the nanoparticles. In support of this mechanism are the results that there is no detectable release of nitrite from the particles when they are added to water [8]. However, if there is any nitrite retained in the nanoparticles, when they are exposed to an aqueous solvent, protons enter the nanoparticles and react with nitrite to slowly produce $\mathrm{NO}$ which then diffuses out of the particles. The protonation driven mechanism is supported by results presented in Figure $1 \mathrm{C}$, where it can be seen that at low $\mathrm{pH}$ there is a greater amount of $\mathrm{NO}$ being released at early times. Definitive determination of the mechanism is currently being explored through the use of a variety of spectroscopic tools to probe the nature and fate of nitrite within the nanoparticles as a function of exposure to water.

The NO-np mediated vasorelaxation is independent of NOS inhibition, however the NO released by $10 \mathrm{mg} / \mathrm{kg}_{\mathrm{BW}}$ of NO-np was not sufficient to completely supplant the NO inhibited from the NOS system. The relaxant responses produced by NO-np could not be attributed to nitrite, consistent with earlier in vitro results.[8] The NO-np impact on blood pressure and vessel diameter was diminished by administering ODQ, a selective inhibitor of soluble guanylate cyclase (GC).[24] GC is the enzyme responsible for increasing cyclic guanosine monophosphate (cGMP) levels in response to increasing concentrations of NO and stimulating cGMP-dependent protein kinase (PKG) in the vascular smooth muscle. Inhibition of $\mathrm{NO}$ binding to $\mathrm{GC}$ acts downstream on the $\mathrm{NO}$ action in vasorelaxation, functionally ablating most of the activity of $\mathrm{NO}$ on vascular tone. Thus, ODQ-induced mitigation of the previously demonstrated NO-np vascular impact serves to confirm NO as the active species released from the NO-np.

In addition to regulating vascular tone, $\mathrm{NO}$ and cGMP signaling can also inhibit proliferation and induce apoptosis in vascular smooth muscle.[25] Intracellular cGMP levels are determined by the rate of cGMP hydrolysis as well as synthesis. The major enzymes responsible for degrading cGMP in vascular smooth muscle are cGMP-specific phosphodiesterase (PDE) Type 5 (PDE5) and calcium/calmodulin-dependent PDE Type 1. [17] Infusion of NO-np in animals pretreated with PDE5 inhibitor Zaprinast increased the effects of NO-np on blood pressure and more so on vessel diameter. Thus, acute inhibition of PDE5 activity, by drugs such as sildenafil, combined with NO-np represents an attractive therapeutic strategy to manipulate systemic vascular tone.

There are strong indications that activated macrophages can recognize nanoparticles in the blood.[26] The presence of exogenous NO from the nanoparticles prevented inflammatory reaction to external agents, especially the number of immobilized and rolling leukocytes. As not all macrophages and monocytes are identical[27], macrophages found within microcirculatory regions are more active in cytokine production and have stronger phagocytic receptors.[28] NO-np technology could prevent long-circulating nanoparticles from being trapped by such macrophage populations and this action may be even further enhanced with human macrophages. The NO-np technology seems to be optimal for "macrophage-evasion," providing a long-circulating drug reservoir from which the drug can be released into the vascular compartment in a continuous and controlled manner.

Here we present the physiological responses to intravascular infusion of NO-np, consistent with a sustained release of physiologically relevant levels of NO, reflected by decreased blood pressure and increased vascular relaxation. The NO-np are effective vasorelaxants, whose potency is limited by the dose and time course of hydration. They do not require 
external reducing agents, nor do they generate tolerance during prolonged continuous use. The efficacy and potency of the exogenous NO released by the NO-np is evident from the systemic hypotension and increase in exhaled concentrations of NO. Additionally, NO-np do not exhibit many of the shortcomings of current methods of applying NO in clinical settings, suggesting that it may be a conduit through which the potential of NO can be harnessed and translated into a practical therapeutic strategy. In summation, the presented results indicate that the NO-np platform, for which we have also demonstrated efficacy via topical delivery for the treatment of soft tissue infections,[29] has considerable promise both for therapeutic applications requiring sustained systemic NO delivery, and for basic research focusing on the behavior and activity of intravascular NO. Until now, both topical and injection studies have failed to show any toxicity attributable to the NO-nanoparticles. However, future studies to address biodistribution, clearance, and biocompatibility of the NO releasing nanoparticles are essential to determine the real clinical relevance.

\section{Acknowledgments}

This work was supported by Program project P01 HL071064 and FJC, A Foundation of Philanthropic Funds. A.J.F was supported by research grants from the American Society for Dermatologic Surgery Cutting Edge Program, the La-Roche Posay North American Foundation, and the Women's Dermatologic Society. The authors thank Froilan P. Barra and Cynthia Walser for the surgical preparation of the animals.

\section{References}

1. Zacharia IG, Deen WM. Diffusivity and solubility of nitric oxide in water and saline. Ann Biomed Eng. 2005; 33:214-222. [PubMed: 15771275]

2. Moncada S, Palmer MJ, Higgs EA. Nitric Oxide: Physiology, pathophysiology, and pharmacology. Pharmacol Rev. 1991; 43:109-134. [PubMed: 1852778]

3. Busse R, Fleming I. Pulsatile stretch and shear stress: physical stimuli determining the production of endothelium-derived relaxing factors. J Vasc Res. 1998; 35:73-84. [PubMed: 9588870]

4. Lancaster JR Jr. A tutorial on the diffusibility and reactivity of free nitric oxide. Nitric Oxide. 1997; 1:18-30. [PubMed: 9701041]

5. Ichinose F, Roberts JD Jr, Zapol WM. Inhaled nitric oxide: a selective pulmonary vasodilator: current uses and therapeutic potential. Circulation. 2004; 109:3106-3111. [PubMed: 15226227]

6. Zapol WM. Inhaled nitric oxide. Acta Anaesthesiol Scand Suppl. 1996; 109:81-83. [PubMed: 8901957]

7. Homer K, Wanstall J. In vitro comparison of two NONOates (novel nitric oxide donors) on rat pulmonary arteries. Eur J Pharmacol. 1998; 356:49-57. [PubMed: 9761423]

8. Friedman AJ, Han G, Navati MS, Chacko M, Gunther L, Alfieri A, Friedman JM. Sustained release nitric oxide releasing nanoparticles: characterization of a novel delivery platform based on nitrite containing hydrogel/glass composites. Nitric Oxide. 2008; 19:12-20. [PubMed: 18457680]

9. Maragos CM, Morley D, Wink DA, Dunams TM, Saavedra JE, Hoffman A, Bove AA, Isaac L, Hrabie JA, Keefer LK. Complexes of .NO with nucleophiles as agents for the controlled biological release of nitric oxide. Vasorelaxant effects. J Med Chem. 1991; 34:3242-3247. [PubMed: 1956043]

10. Maragos CM, Wang JM, Hrabie JA, Oppenheim JJ, Keefer LK. Nitric oxide/nucleophile complexes inhibit the in vitro proliferation of A375 melanoma cells via nitric oxide release. Cancer Res. 1993; 53:564-568. [PubMed: 8425188]

11. Homer KL, Wanstall JC. Cyclic GMP-independent relaxation of rat pulmonary artery by spermine NONOate, a diazeniumdiolate nitric oxide donor. Br J Pharmacol. 2000; 131:673-682. [PubMed: 11030715]

12. Endrich B, Asaishi K, Götz A, Messmer K. Technical report: A new chamber technique for microvascular studies in unanaesthetized hamsters. Res Exp Med. 1980; 177:125-134.

13. Sakata M, Yoshida A, Haga M. Methemoglobin in blood as determined by double-wavelength spectrophotometry. Clin Chem. 1982; 28:508-511. [PubMed: 7067094] 
14. Childs EW, Udobi KF, Wood JG, Hunter FA, Smalley DM, Cheung LY. In vivo visualization of reactive oxidants and leukocyte-endothelial adherence following hemorrhagic shock. Shock. 2002; 18:423-427. [PubMed: 12412620]

15. Tapec R, Zhao XJ, Tan W. Development of organic dye-doped silica nanoparticles for bioanalysis and biosensors. J Nanosci Nanotechnol. 2002; 2:405-409. [PubMed: 12908270]

16. Schrammel A, Behrends S, Schmidt K, Koesling D, Mayer B. Characterization of $1 \mathrm{H}-$ [1,2,4] oxadiazolo[4,3-a]quinoxalin-1-one as a heme-site inhibitor of nitric oxide-sensitive guanylyl cyclase. Mol Pharmacol. 1996; 50:1-5. [PubMed: 8700100]

17. Rybalkin SD, Yan C, Bornfeldt KE, Beavo JA. Cyclic GMP phosphodiesterases and regulation of smooth muscle function. Circ Res. 2003; 93:280-291. [PubMed: 12933699]

18. Allman KG, Young JD, Carapiet D, Stevens JE, Ostman-Smith I, Archer LN. Effects of oxygen and nitric oxide in oxygen on pulmonary arterial pressures of children with congenital cardiac defects. Pediatr Cardiol. 1996; 17:246-250. [PubMed: 8662048]

19. Wanstall JC, Kaye JA, Gambino A. The in vitro pulmonary vascular effects of FK409 (nitric oxide donor): a study in normotensive and pulmonary hypertensive rats. Br J Pharmacol. 1997; 121:280286. [PubMed: 9154338]

20. Ray A, Friedman BA, Friedman JM. Trehalose glass-facilitated thermal reduction of metmyoglobin and methemoglobin. J Am Chem Soc. 2002; 124:7270-7271. [PubMed: 12071726]

21. Navati MS, Friedman JM. Sugar-derived glasses support thermal and photo-initiated electron transfer processes over macroscopic distances. J Biol Chem. 2006; 281:36021-36028. [PubMed: 17005567]

22. Navati MS, Friedman JM. Glass matrix-facilitated thermal reduction: a tool for probing reactions of met hemoglobin with nitrite and nitric oxide. J Phys Chem B. 2010; 114:2938-2943. [PubMed: 20146537]

23. Navati MS, Friedman JM. Reactivity of glass-embedded met hemoglobin derivatives toward external NO: implications for nitrite-mediated production of bioactive NO. J Am Chem Soc. 2009; 131:12273-12279. [PubMed: 19663497]

24. Garthwaite J, Southam E, Boulton CL, Nielsen EB, Schmidt K, Mayer B. Potent and selective inhibition of nitric oxide-sensitive guanylyl cyclase by $1 \mathrm{H}-[1,2,4]$ oxadiazolo[4,3-a]quinoxalin-1one. Mol Pharmacol. 1995; 48:184-188. [PubMed: 7544433]

25. Pollman MJ, Yamada T, Horiuchi M, Gibbons GH. Vasoactive substances regulate vascular smooth muscle cell apoptosis. Countervailing influences of nitric oxide and angiotensin II. Circ Res. 1996; 79:748-756. [PubMed: 8831498]

26. Moghimi SM, Gray T. A single dose of intravenously injected poloxamine-coated long-circulating particles triggers macrophage clearance of subsequent doses in rats. Clin Sci (Lond). 1997; 93:371-379. [PubMed: 9404230]

27. Rutherford MS, Witsell A, Schook LB. Mechanisms generating functionally heterogeneous macrophages: chaos revisited. J Leukoc Biol. 1993; 53:602-618. [PubMed: 8501399]

28. Ito S, Naito M, Kobayashi Y, Takatsuka H, Jiang S, Usuda H, Umezu H, Hasegawa G, Arakawa M, Shultz LD, Elomaa O, Tryggvason K. Roles of a macrophage receptor with collagenous structure (MARCO) in host defense and heterogeneity of splenic marginal zone macrophages. Arch Histol Cytol. 1999; 62:83-95. [PubMed: 10223745]

29. Martinez LR, Han G, Chacko M, Mihu MR, Jacobson M, Gialanella P, Friedman AJ, Nosanchuk JD, Friedman JM. Antimicrobial and Healing Efficacy of Sustained Release Nitric Oxide Nanoparticles Against Staphylococcus Aureus Skin Infection. J Invest Dermatol. 2009 
a)

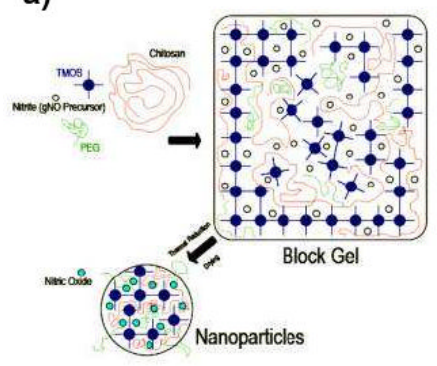

b)

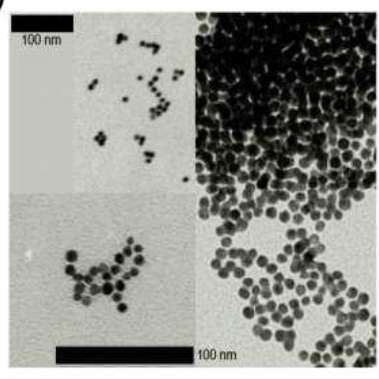

c)

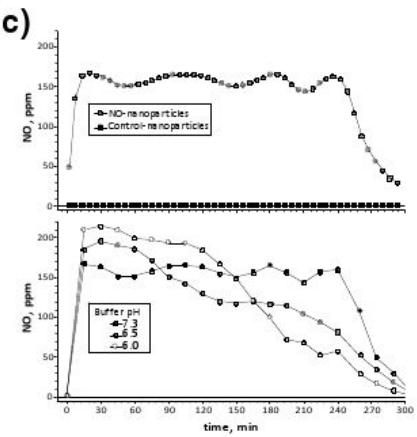

Figure 1. NO releasing nanoparticle synthesis and morphology

a) Schematic of NO releasing nanoparticle synthesis. The various components are prepared and combined to form a block gel consisting of a silica hydrogel matrix encompassing the other ingredients. The block gel is dried and the nitrite precursor converted to NO via thermal reduction, resulting in the formation of the nanoparticles. b) TEM of NO releasing nanoparticles. The scale bars represent 100nm, the bottom one representing the lower left and right panes and the upper one for the upper left image. c) NO gas levels were measured using a chemiluminescent $\mathrm{NO}$ analyzer. Upper panel, $1 \mathrm{mg}$ of NO-np or Control-np at 7.4 $\mathrm{pH}$. Lower panel, $1 \mathrm{mg}$ of NO-np at $6.0,6.5$ and $7.3 \mathrm{pH}$. 


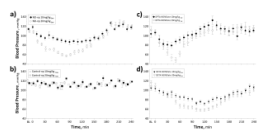

Figure 2. Effects in blood pressure after infusion of NO releasing nanoparticles (NO-np), control nanoparticles (Control-np) and NONOates donors

a) Infusion of $10 \mathrm{mg} / \mathrm{kg}(\mathrm{n}=5) \mathrm{NO}-\mathrm{np}$ and $20 \mathrm{mg} / \mathrm{kg}(\mathrm{n}=5)$ NO-np. Maximal decrease pressure was measured 90 min after infusion. MetHb levels $4 \mathrm{~h}$ after infusion of NO-np were $9 \pm 2 \%$ for $10 \mathrm{mg} / \mathrm{kg}$ and $14 \pm 3 \%$ for $20 \mathrm{mg} / \mathrm{kg}$, respectively. b) Infusion of $10 \mathrm{mg} / \mathrm{kg}$ $(\mathrm{n}=5)$ Control-np and $20 \mathrm{mg} / \mathrm{kg}(\mathrm{n}=5)$ Control-np. No changes in blood pressure were measure after in fusion of Control-np. c) Infusion of $10 \mathrm{mg} / \mathrm{kg}(\mathrm{n}=5)$ DPTA NONOate and $20 \mathrm{mg} / \mathrm{kg}(\mathrm{n}=5)$ DPTA NONOate. d) Infusion of $10 \mathrm{mg} / \mathrm{kg}(\mathrm{n}=5)$ DETA NONOate and 20 $\mathrm{mg} / \mathrm{kg}(\mathrm{n}=5)$ DETA NONOate. 


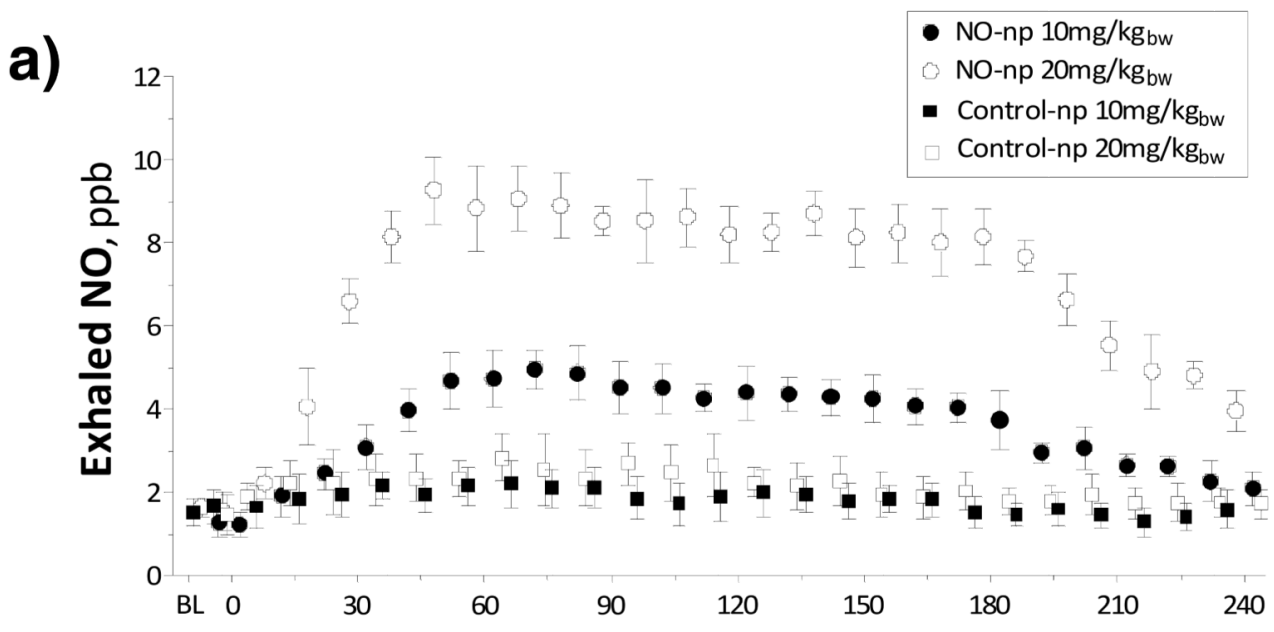

b)

Time, $\min$

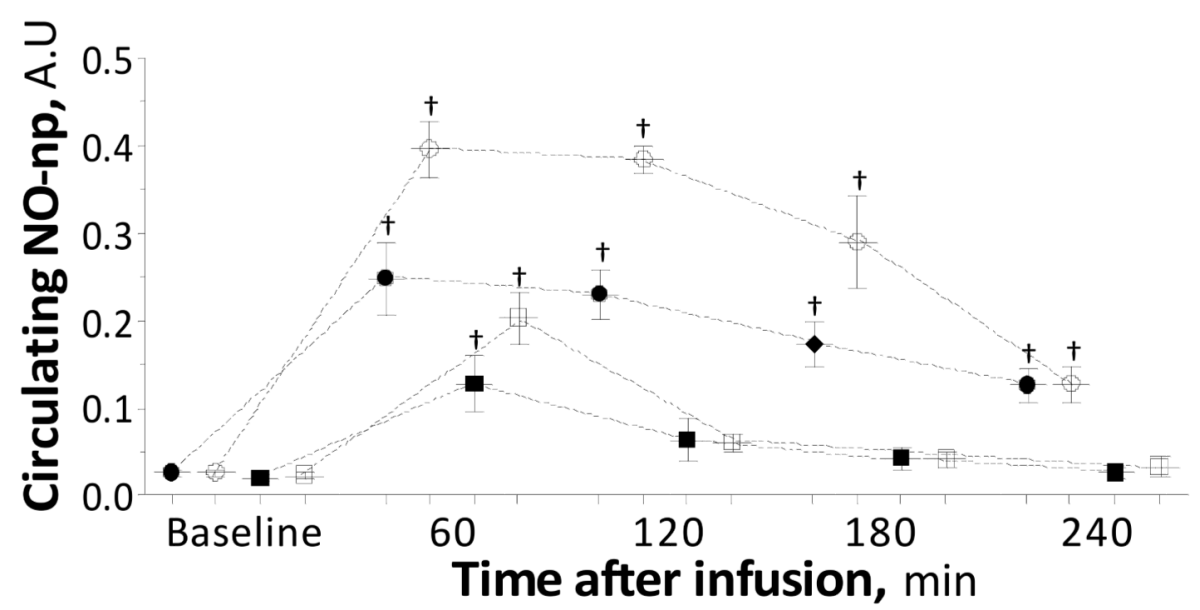

Figure 3. Exhaled NO after infusion of NO releasing nanoparticles (NO-np) and control nanoparticles (Control-np)

a) Exhaled NO after infusion of $10 \mathrm{mg} / \mathrm{kg}$ and $20 \mathrm{mg} / \mathrm{kg}(\mathrm{n}=5)$ of NO-np and Control-np. b) Intravascular fluorescence of NO-np and Control-np. $\dagger, \mathrm{P}<0.05$ to Baseline. 


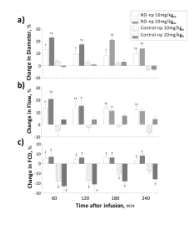

Figure 4. Microvascular changes and blood chemistry after infusion of NO releasing nanoparticles (NO-np) and control nanoparticles (Control-np)

a) Changes in arteriolar diameter after infusion of 10 (white bars) and 20 (gray bars) mg/kg of NO-np (no pattern) and Control-np (pattern upward diagonal). Diameters ( $\mu \mathrm{m}$, mean \pm $\mathrm{SD})$ at baseline were $61.7 \pm 7.9, \mathrm{~N}=68 . \mathrm{N}=$ number of vessels studied. $\uparrow, \mathrm{P}<0.05$ to

Baseline; $\ddagger, \mathrm{P}<0.05$ to Control-np at same concentration. b) Changes in arteriolar blood flow after infusion of NO-np and Control-np. Flow (nl/s, mean \pm SD) at baseline were 12.6 \pm 3.8 , $\mathrm{N}=68$. $\dagger, \mathrm{P}<0.05$ to Baseline; $\ddagger, \mathrm{P}<0.05$ to Control-np at same concentration. c) Changes in functional capillary density (FCD) after infusion of NO-np and Control-np. FCD (perfused capillaries $/ \mathrm{cm}^{-1}$, mean $\pm \mathrm{SD}$ ) at baseline were $106 \pm 6, \mathrm{n}=20 . \dagger, \mathrm{P}<0.05$ to Baseline; $\ddagger$, $\mathrm{P}<0.05$ to Control-np at same concentration. 

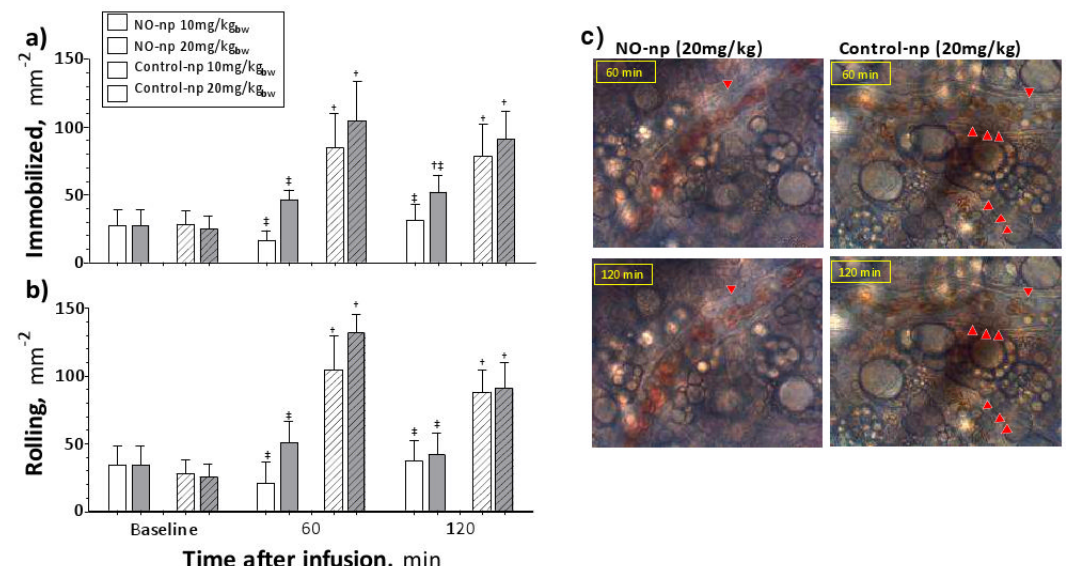

Figure 5. Rolling and immobilized leukocytes after infusion of NO releasing nanoparticles (NOnp) and control nanoparticles (Control-np)

a) Immobilized leukocytes after infusion of 10 (white bars) and 20 (gray bars) mg/kg of NO$\mathrm{np}$ (no pattern) and Control-np (pattern upward diagonal). 6 animals were used in each group, $6-8$ venules were selected in each animal. $\uparrow, \mathrm{P}<0.05$ to Baseline; $\ddagger, \mathrm{P}<0.05$ to Control-np at same concentration. b) Rolling leukocytes after infusion of NO-np and Control-np. Rolling leukocytes were quantified in the same animals and locations as Immobilized. $\dagger, \mathrm{P}<0.05$ to Baseline; $\ddagger, \mathrm{P}<0.05$ to Control-np at same concentration. c) Immobilized and rolling leukocytes after infusion of $20 \mathrm{mg} / \mathrm{kg}$ of NO-np and Control-np. Red arrows point to immobilized leukocytes on the images, other leukocytes in the images are rolling of flowing. 

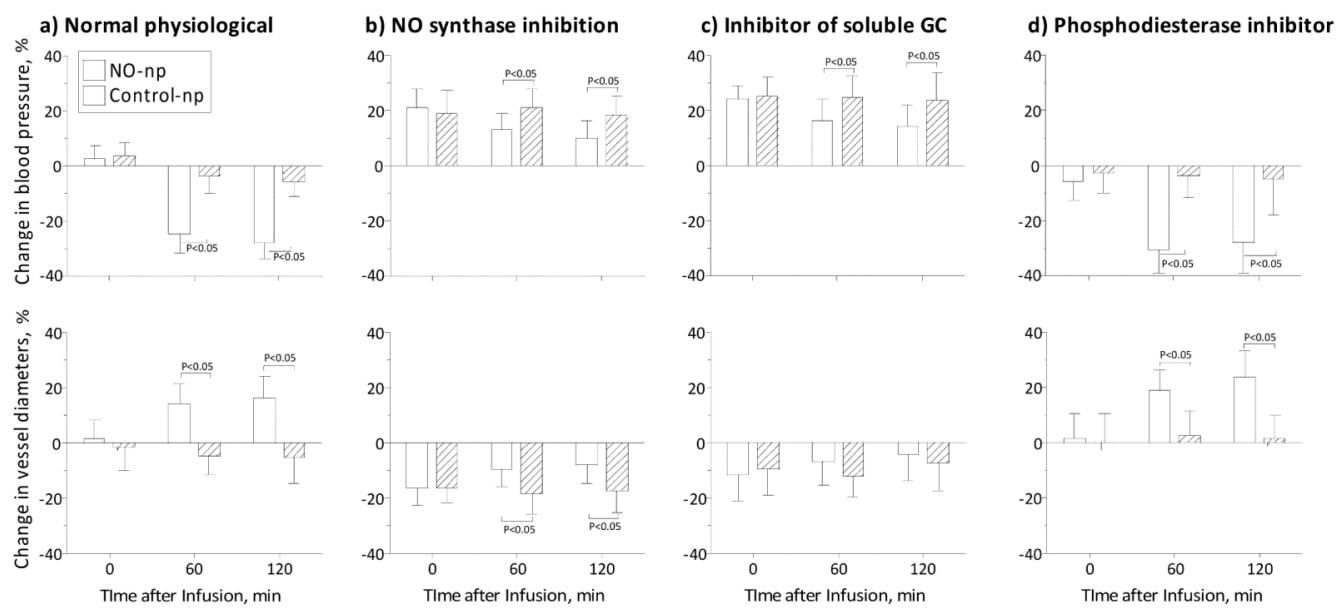

Figure 6. Blood pressure vascular tone effects of NO-np and Control-np mediated by NO synthase (NOS) and guanylate cyclase (GCM) metabolism

a) Infusion of $10 \mathrm{mg} / \mathrm{kg}$ of NO-np $(\mathrm{n}=5)$ and Control-np $(\mathrm{n}=5)$ during normal physiological condition. Blood pressure $(\mathrm{mmHg}$, mean $\pm \mathrm{SD})$ at baseline for each group were NO-np: 112 $\pm 9(n=5)$ and Control-np: $114 \pm 8(n=5)$. Diameters $(\mu \mathrm{m}$, mean \pm SD) at baseline for each group were NO-np: $62 \pm 10(\mathrm{~N}=24)$ and Control-np: $64 \pm 12(\mathrm{~N}=24)$. b) Infusion of $10 \mathrm{mg} /$ $\mathrm{kg}$ of NO-np $(\mathrm{n}=5)$ and Control-np $(\mathrm{n}=5)$ during NO synthase inhibition with L-NAME. Blood pressure $(\mathrm{mmHg}$, mean $\pm \mathrm{SD})$ at baseline for each group were NO-np: $111 \pm 8(\mathrm{n}=5)$ and Control-np: $110 \pm 7(\mathrm{n}=5)$. Diameters $(\mu \mathrm{m}$, mean \pm SD) at baseline for each group were NO-np: $60 \pm 12$ (N=22) and Control-np: $65 \pm 8(\mathrm{~N}=20)$. c) Infusion of $10 \mathrm{mg} / \mathrm{kg}$ of NO-np $(\mathrm{n}=5)$ and Control-np $(\mathrm{n}=5)$ after ODQ. Blood pressure $(\mathrm{mmHg}$, mean \pm SD) at baseline for each group were NO-np: $110 \pm 6(n=5)$ and Control-np: $112 \pm 7(n=5)$. Diameters $(\mu \mathrm{m}$, mean $\pm \mathrm{SD})$ at baseline for each group were NO-np: $58 \pm 11(\mathrm{~N}=26)$ and Control-np: $62 \pm$ $10(\mathrm{~N}=20)$. d) Infusion of $10 \mathrm{mg} / \mathrm{kg}$ of NO-np $(\mathrm{n}=5)$ and Control-np $(\mathrm{n}=5)$ during phosphodiesterase inhibitor Zaprinast. Blood pressure $(\mathrm{mmHg}$, mean $\pm \mathrm{SD})$ at baseline for each group were NO-np: $109 \pm 7(\mathrm{n}=5)$ and Control-np: $112 \pm 6(\mathrm{n}=5)$. Diameters $(\mu \mathrm{m}$, mean \pm SD) at baseline for each group were NO-np: $64 \pm 12(\mathrm{~N}=20)$ and Control-np: $66 \pm$ $10(\mathrm{~N}=20)$. 


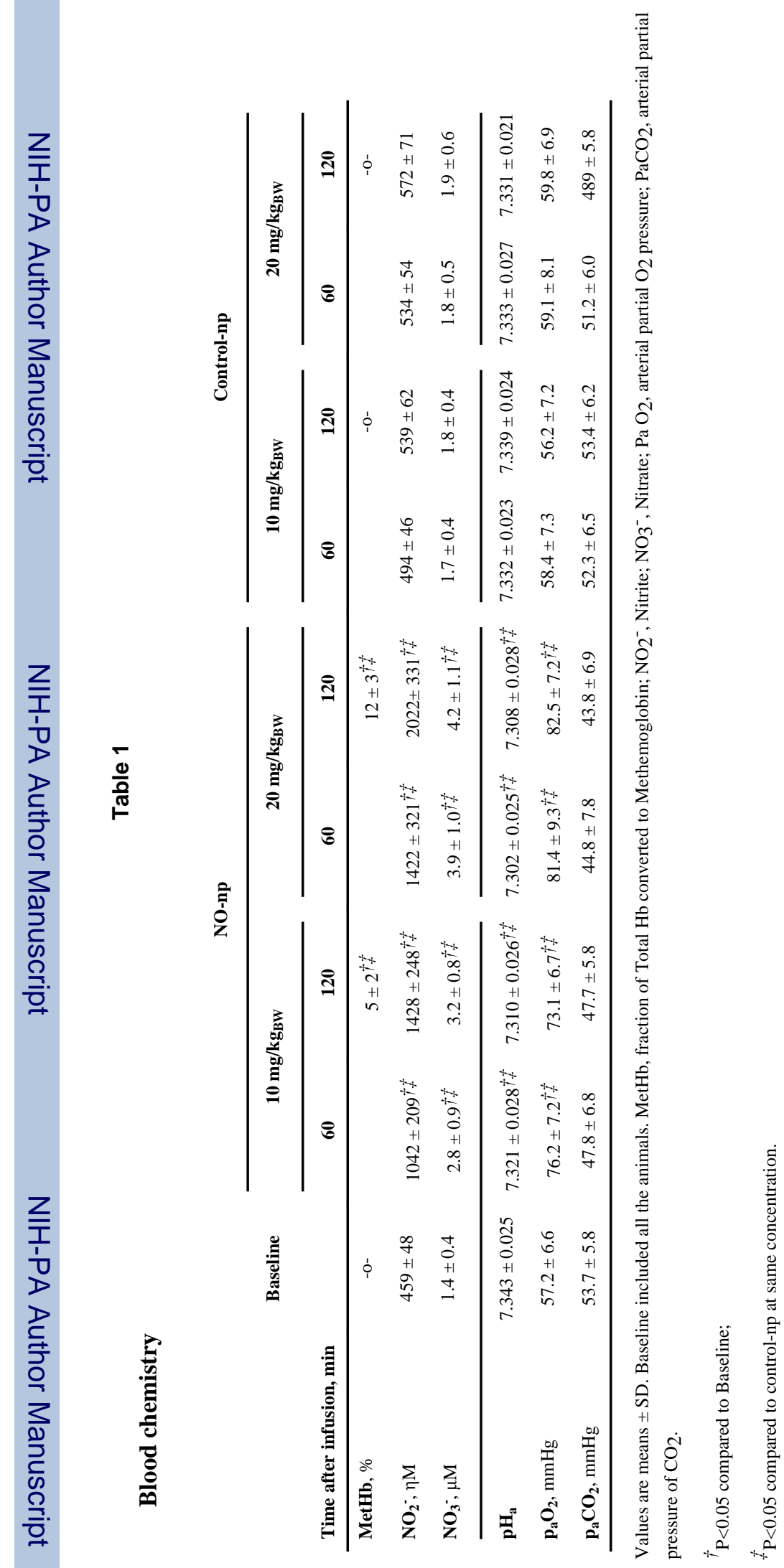

